\title{
Attentional Control in Anterior Cingulate Cortex Based on Probabilistic Cueing
}

\author{
Esther Aarts and Ardi Roelofs
}

\begin{abstract}
In Stroop-like tasks, conflict effects in behavioral measures and ACC activity are smaller on trials following an incongruent trial than following a congruent one. Researchers have found no agreement on whether these sequential effects in ACC can be driven by experienced incongruency only or also by expectations about target types. In the present fMRI experiment, we specifically manipulated the expectancies by using symbolic cues predicting with $75 \%$ or $50 \%$ certainty the incongruent or congru-
\end{abstract}

\section{INTRODUCTION}

Attentional control includes the ability to act according to goals in the face of distraction. This ability is critical to normal human functioning and it is a hallmark of general intelligence (Duncan, 1995, 2005). The Stroop conflict task (Stroop, 1935) is often used to study attentional control. In a commonly used version of this task, participants name the ink color of written color words (MacLeod, 1991). Stimuli can be congruent or incongruent (e.g., the word RED in red or green ink, respectively). RT is typically longer on incongruent than on congruent trials, referred to as the "conflict effect." Neuroimaging studies have shown that ACC is implicated in attentional control, together with other areas in frontal and parietal cortex (Paus, 2001; Bush, Luu, \& Posner, 2000; Miller, 2000; Picard \& Strick, 1996).

Conflict effects in task performance are generally found to be smaller on trials following an incongruent than a congruent trial (for a review, see Egner, 2007). The prevalent interpretation of this sequential effect is that it reflects conflict-induced adjustments in attentional control (Botvinick, Braver, Barch, Carter, \& Cohen, 2001). fMRI studies have demonstrated the involvement of ACC in these adjustments (e.g., Kerns et al., 2004; Botvinick, Nystrom, Fissell, Carter, \& Cohen, 1999). According to a prominent view, conflict on incongruent trials is detected by ACC, which subsequently signals to dorsolateral prefrontal cortex (DLPFC) the need to up-regulate attentional control, which implies a greater focus on the target dimension (e.g., the color of a Stroop stimulus). There will be no such control adjustment on congruent trials. Consequently, control will be

Radboud University Nijmegen, The Netherlands ent targets in a Stroop-like task. Both behavioral and dorsal ACC data replicated previous sequential effects, with conflict effects being smallest for targets following the cues that predicted with 75\% certainty the incongruent targets. However, these effects were not driven by experienced conflict but by symbolic cues. These results demonstrate differential attentional control activity in ACC after probabilistic cueing, providing evidence for control adjustments driven by changes in expectation. lower (i.e., attention will be less focused) following congruent than incongruent trials, and the conflict effect in ACC activity and RTs will be larger following experienced congruency than incongruency (Botvinick, Cohen, \& Carter, 2004).

However, control adjustments in ACC do not only have to take place on the basis of experienced conflict. For example, fMRI studies have found cue-related ACC activity associated with an allocation of attention to taskrelevant information (Orr \& Weissman, 2009; Weissman, Gopalakrishnan, Hazlett, \& Woldorff, 2005). Recently, we demonstrated that cue-based adjustments of attentional control are associated with ACC activity in Stroop-like tasks as well (Aarts, Roelofs, \& van Turennout, 2008). In an fMRI study, cues were $100 \%$ predictive of the upcoming Stroop-like target conditions. Compared with nonpredictive cues, predictive cues elicited ACC activity. Importantly, ACC activity was equally enhanced for congruent- and incongruent-predicting cues, and performance was improved after both types of informative cues. This suggests that cue-based control in ACC may be independent of upcoming response conflict. Critically, a conflict effect in target-related ACC activity was observed following nonpredictive cues but not following 100\%-valid congruent-predicting and incongruentpredicting cues, even though the RTs still exhibited a conflict effect. Cue-related ACC activity correlated with activity in premotor areas, which suggests that the control adjustments were made, at least partly, in premotor cortex (Aarts et al., 2008).

The abovementioned evidence indicates that attentional adjustments can be made in response to predictive cues, that is, "proactively" rather than "reactively" 
(i.e., in response to targets) only. Could these proactive control adjustments also give rise to the reactive effects observed when studying trial-to-trial sequential effects in conflict tasks? Behaviorally, it has indeed been shown that conflict effects are greater following probabilistic (i.e., partly valid) cues that predict an upcoming congruent target than following probabilistic cues that predict an incongruent target (Gratton, Coles, \& Donchin, 1992). However, it has not been demonstrated yet that these proactive control adjustments also give rise to targetrelated effects in ACC paralleling those observed in studies investigating sequential effects as a function of experienced conflict.

In our previous fMRI study with a cued Stroop-like task (Aarts et al., 2008), we could not investigate conflict effects on congruent and incongruent targets following the different kinds of predictive cues because the cues were 100\% valid, hence, were always followed by the same target type. To investigate the effect of cue-induced (as opposed to conflict-induced) control adjustments on the Stroop conflict effect, the present experiment used cues that predicted the upcoming Stroop target with 75\% validity. This way, congruent and incongruent targets could follow both congruent- and incongruent-predicting cues, which corresponds to earlier studies investigating trial-to-trial adjustments. Of course, a condition-cued Stroop study might not be completely comparable to studying sequential effects in a "normal" Stroop study without cues. For example, to have participants engage in a certain strategy in the present study, a congruent-predicting cue could only be followed by an incongruent target in $25 \%$ of the times, and vice versa for incongruent-predicting cues. This is, however, still quite similar to what is sometimes done in Stroop studies investigating trial-to-trial adjustments in ACC. For example, one of the key studies had $70 \%$ congruent trials to increase conflict effects (Kerns et al., 2004). Thus, in that study, only 30\% of the congruent trials were followed by an incongruent trial, which is comparable to the probabilities in the present study.

How can symbolic cues induce similar effects as a previous trial on the upcoming Stroop target? We hypothesize that control adjustments can be driven not only by experienced conflict on incongruent trials but also by expectations concerning target types (cf. Gratton et al., 1992; Logan \& Zbrodoff, 1979, 1982). The expectation of a congruent stimulus leads to a widening of attention, so that the facilitatory influence from a congruent distractor on response selection is increased. In principle, participants may correspondingly narrow attention (i.e., increase focus on the relevant dimension) if an incongruent stimulus is expected, so that the interference from the distractor will be reduced. However, past research demonstrated that participants' tendency to widen attention when expecting congruent trials is greater than their tendency to narrow attention when expecting incongruent trials (Gratton et al., 1992; Logan \& Zbrodoff, 1979, 1982). Either way, attention will be wider following congruent- than incongruent- predicting cues and, consequently, the conflict effect in ACC activity and RTs will be larger following expected congruency than incongruency, as empirically observed. This is because a widening of attention is beneficial on congruent trials but detrimental on incongruent trials. These attentional adjustments parallel the adjustments hypothesized by the conflict-based account of sequential effects (Botvinick et al., 2004). However, the main difference between the theoretical accounts is the cause of the adjustments: experienced conflict versus expectancies induced by symbolic cues.

Elsewhere, we and others (De Pisapia \& Braver, 2006) hypothesized that ACC activity reflects both proactive control processes (Aarts et al., 2008) and reactive control processes (Roelofs, van Turennout, \& Coles, 2006). We proposed that reactive control involves boosting the activation of the response to the relevant dimension among competing alternatives (Roelofs et al., 2006). Moreover, we proposed that proactive control involves adjustments of the connections between the relevant dimensions and distractor dimensions, on the one hand, and the associated responses, on the other (Aarts et al., 2008), sometimes referred to as attentional "gating" (Wuhr \& Kunde, 2008). An informative cue preceding an incongruent target might encourage participants to strengthen the connections between the relevant dimension and their responses (i.e., "focusing" of attention) because the distractors elicit the wrong response. However, an informative cue preceding a congruent target might encourage participants to strengthen the connections between the distractors and the corresponding responses (i.e., "widening" of attention) because the distractors also elicit the correct response. In our previous study with 100\%-valid cues, ACC activity was enhanced for both congruent- and incongruentpredicting cues and both types of cues improved performance. This suggests that congruent-predicting cues indeed induce a widening of attention and that incongruentpredicting cues can lead to attention narrowing (Aarts et al., 2008).

Our previous data (Aarts et al., 2008) suggest that if control can be optimized proactively in preparation for the target (based on 100\%-valid cues), control processes are no longer triggered reactively by the target, which explains why ACC was active in response to the cue but not to the target. That is, with 100\%-valid cues, there are only benefits of cueing, because the attention strategy can be optimal for the cued target type. However, with probabilistic cueing, attention cannot be optimally set: Wide attention after congruent-predicting cues hampers performance to invalidly cued incongruent targets, and narrow attention after incongruent-predicting cues unnecessarily delays responding to invalidly cued congruent targets. This predicts differences in conflict-related ACC activity at the targets depending on the preceding cue type in the present experiment, paralleling the effects that are obtained as a function of a preceding trial (Kerns et al., 2004; Botvinick et al., 1999). 


\section{METHODS}

\section{Subjects}

Twenty neurologically healthy Dutch undergraduates (12 women and 8 men, mean age $=21.2$ years, range $=$ 19-26 years) participated in the experiment. All participants were right-handed and native speakers of Dutch. They were compensated for participation and gave written informed consent in a manner approved by the Dutch Central Committee on Research Involving Human Subjects (CCMO).

\section{Stimuli and Paradigm}

The participants were scanned while performing a manual arrow-word version of the Stroop task. As with color-word Stroop stimuli, responding in this task is usually slower on incongruent than on congruent trials (Roelofs et al., 2006; Turken \& Swick, 1999; Baldo, Shimamura, \& Prinzmetal, 1998). The targets consisted of written words in arrows (Figure 1). The lines and letters of the targets were white on a black background. The arrows pointed to the left or to the right. The word in the arrow was the Dutch word for right (rechts) or for left (links). Participants responded manually to the words by pressing a left or right button on a scanner-compatible button box. Participants were told to respond as quickly and accurately as possible with the left middle finger (for left response) and the left index finger (for right response). In the congruent target condition, the arrow and the word denoted the same direction (e.g., the word right in an arrow pointing to the right). In the incongruent target condition, the arrow and the word denoted a different direction (e.g., the word left in an arrow pointing to the right).

Every target was preceded by a cue (see Figure 1). The cue was a colored square. A red cue predicted with $75 \%$

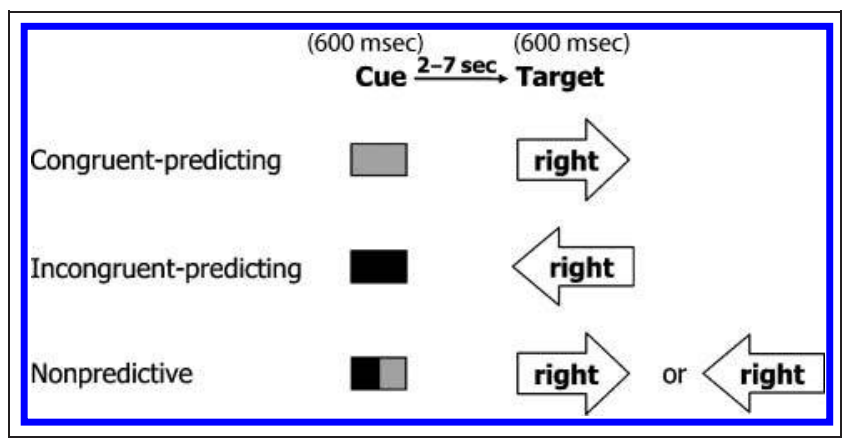

Figure 1. Experimental paradigm. Depicted are the predictive and nonpredictive cues and examples of congruent and incongruent targets with the word "right." Dark green cues (here: gray) were followed by congruent targets with 75\% chance, and red cues (here: black) were followed by incongruent targets with 75\% chance. Combined red-green cues (here: gray-black) predicted one of the target conditions with 50\% chance, and hence were uninformative. The task was to manually respond to the word. In the actual experiment, targets consisted of white lines and letters on a black background. certainty that the upcoming target would be incongruent. A green cue predicted with $75 \%$ certainty that the upcoming target would be congruent. A combined red-green cue predicted with 50\% certainty either one of the upcoming targets, and thus, was uninformative. Participants were told to pay explicit attention to the cues because they would be informative about the upcoming target. It was brought to the participants' attention that during congruent trials one could be helped by the nonrelevant dimension of the target (i.e., the arrow) and that one should not be distracted by the arrow in case of an incongruent trial (cf. Gratton et al., 1992; Logan \& Zbrodoff, 1982). The experiment included 350 trials, consisting of 140 incongruent-predicting, 140 congruent-predicting, and 70 nonpredictive cues. The 140 incongruent-predicting cues were followed by 105 incongruent Stroop targets and 35 congruent Stroop targets. The 140 congruent-predicting cues were followed by 105 congruent Stroop targets and 35 incongruent Stroop targets. The 70 nonpredictive cues were followed by 35 congruent and 35 incongruent Stroop targets. Cue and target types were randomly intermixed.

Cues and targets remained on the screen for 600 msec. The target followed the cue after a variable delay of $2-7 \mathrm{sec}$. There was a similar variable delay of $2-7$ sec between a target and the next cue. Thirty-five null events of $10 \mathrm{sec}$ were also used. Separately for each participant, an optimal random sequence of trials with optimal variable delays was calculated with a simulation of the BOLD response in SPM99 (Wellcome Department of Cognitive Neurology, London). Hence, we could reliably distinguish the BOLD response to the cue from the BOLD response to the target (Josephs, Turner, \& Friston, 1997). Because the delay between cue and target could not be predicted, the participant needed to be ready to respond at any time.

\section{Functional Imaging}

Whole-brain imaging was performed on a 3-Tesla MR scanner (Magnetom Trio, Siemens Medical Systems, Erlangen, Germany). Functional data were acquired using a gradientecho echo-planar scanning sequence (repetition time = $2100 \mathrm{msec}$, echo time $=30 \mathrm{msec}, 33$ axial slices, voxel size $=3.5 \times 3.5 \times 3.5 \mathrm{~mm}$, field of view $=224 \mathrm{~mm}$, flip angle $=70^{\circ}$ ). All functional images were acquired in a single run lasting $55 \mathrm{~min}$. Visual stimuli were projected on a screen and were viewed through a mirror attached to the head coil. After the acquisition of functional images, a high-resolution anatomical scan (T1-weighted MP-RAGE, 192 slices) was obtained.

\section{Behavioral Data Analysis}

The mean RTs of the correct manual responses and the error rates were analyzed using repeated measures ANOVAs with the factors target condition (congruent, incongruent) and cue condition (congruent-predicting, incongruentpredicting, nonpredictive). All variables were tested within 
participants. Specific effects were tested with paired $t$ tests. An effect was called significant when $p<.05$.

\section{fMRI Data Analysis}

fMRI data were analyzed with BrainVoyager QX (Brain Innovation, Maastricht, The Netherlands). Functional images were corrected for slice time acquisition (using sinc interpolation) and 3-D motion correction was performed to detect and correct for small head movements. Linear trend removal was performed and the signal was temporal high-pass filtered to remove low-frequency nonlinear drifts of three or fewer cycles per time course. Functional images were coregistered with the anatomical scan and transformed into Talairach coordinate space using the nineparameter landmark method of Talairach and Tournoux (1988). Images were spatially smoothed with a full width at half maximum Gaussian kernel of $6 \mathrm{~mm}$.

Statistical analyses were performed in the context of the general linear model, including the following event types of interest: congruent-predicting, incongruent-predicting, and nonpredictive cues; congruent targets preceded by valid, invalid, and nonpredictive cues; and incongruent targets preceded by valid, invalid, and nonpredictive cues. Trials on which participants had made an error were put together as a separate event type of noninterest. Furthermore, six motion parameters were included as event types of noninterest. The event types were modeled with a twogamma hemodynamic response function that was adjusted in such a way that it equaled the hemodynamic response function in SPM99 on the basis of which the jitter was calculated (see above). Random effects group analyses were performed enabling generalization of the statistical inferences to the population level. The statistical threshold for the group analyses was set at $p<.05$, false discovery rate corrected for multiple comparisons (Genovese, Lazar, \& Nichols, 2002).

To further investigate effects in the regions of interest (ROIs), namely, ACC and left and right DLPFC, we obtained subject-averaged beta-weights (i.e., regression coefficients) for all target conditions as indices of effect size for all voxels in the functionally defined ROIs showing an effect of incongruent versus congruent targets preceded by congruentpredicting cues in the random effects group analysis. Using this contrast, we expected to find the greatest targetrelated differences on the basis of previous literature (Kerns et al., 2004; Botvinick et al., 1999). To investigate the effects of cue information in this region, subject averaged beta-weights were extracted for all cue conditions from the voxels in the same ROI showing an effect of incongruent versus congruent targets preceded by congruentpredicting cues in the random effects group analysis. To have less biased beta-weights, we also selected ROIs in ACC and DLPFC on the basis of the more general contrast of incongruent versus congruent targets following all cue types (congruent-predicting, incongruent-predicting, and nonpredictive cues), compared to the very specific contrast involving only one preceding cue type, on a more lenient threshold of $p<.005$, uncorrected for multiple comparisons. To investigate cue-related effects in unbiased ROIs, we used the ROIs from Aarts et al. (2008) in ACC and left DLPFC that showed an effect of informative (100\% predictive) versus nonpredictive (uninformative) cues. For all ROIs, the regional-specific time courses were standardized so that beta-weights reflected the BOLD response amplitude of one condition relative to the variability of the signal. Regionally averaged beta-weights were analyzed in repeated measure ANOVAs. Specific effects were tested by applying paired $t$-contrasts to the beta-weights obtained for the different event types. An effect was called significant when $p<.05$.

\section{RESULTS}

\section{Behavioral Results}

During scanning, we measured RTs and errors of the button presses to the targets following the incongruent-predicting cues (75\% incongruent, 25\% congruent targets), following the congruent-predicting cues (75\% congruent, 25\% incongruent targets), and following the nonpredictive cues (50\% incongruent, 50\% congruent targets). Statistics of the behavioral results are mentioned in Table 1.

\section{Reaction Times}

RTs showed a main effect of target condition, no main effect of cue condition, but a significant interaction of cue condition and target condition (Figure 2A). There was a conflict effect in the RTs following all three cue types. Compared with nonpredictive cues, the conflict effect decreased after incongruent-predicting cues and increased after congruentpredicting cues (Figure 2A and Table 1).

The RTs revealed effects of valid cueing (Table 1). RTs were shorter for congruent targets preceded by valid congruent-predicting cues than by nonpredictive cues. Similarly, RTs tended to be shorter for incongruent targets preceded by valid incongruent-predicting cues than by nonpredictive cues. RTs also revealed effects of invalid cueing. RTs were longer when congruent targets were preceded by invalid incongruent-predicting cues than by nonpredictive cues. Similarly, RTs were longer when incongruent targets were preceded by invalid congruent-predicting cues than when preceded by nonpredictive cues.

\section{Error Rates}

The error rates showed main effects of target and cue condition, and the effects interacted (Figure 2B). As with the RTs, the conflict effect in the error rates was larger after congruent-predicting than incongruent-predicting cues. The conflict effect was marginally larger after nonpredictive than incongruent-predicting cues, and there was a larger 
Table 1. Target-related Effects in Performance and ACC and DLPFC ROIs

\begin{tabular}{|c|c|c|c|c|c|c|c|c|c|}
\hline & $(d f)$ & $R T$ & $E R R$ & $A C C^{a}$ & $A C C^{b}$ & $l D L P F C^{a}$ & $r D L P F C^{a}$ & $l D L P F C^{b}$ & $r D L P F C^{b}$ \\
\hline Main target & $F(1,19)$ & $92.43 * * *$ & $15.75 * * *$ & $7.31 * *$ & $13.11 * *$ & $7.81 * *$ & $11.04 * * *$ & $14.55 * * *$ & $15.72 * * *$ \\
\hline Main cue & $F(2,38)$ & $<1$ & $6.02 * * *$ & $<1$ & 1.16 & $3.25 * *$ & $<1$ & $4.72 * *$ & $<1$ \\
\hline Cue $\times$ Target & $F(2,38)$ & $9.65 * * *$ & $5.42 * *$ & $5.98 * *$ & $2.58^{*}$ & $7.98 * * *$ & $7.62 * * *$ & $6.11 * * *$ & $6.33^{* * * *}$ \\
\hline \multicolumn{10}{|l|}{ Stroop Conflict Effects } \\
\hline INC-CON after con & $t(19)$ & $7.48 * * *$ & $3.90 * * *$ & $4.94 * * *$ & $4.40 * * *$ & $5.06 * * *$ & $6.43 * * *$ & $4.05 * * *$ & $5.74 * * *$ \\
\hline INC-CON after non & $t(19)$ & $7.86 * * *$ & $3.88 * * *$ & $<1$ & 1.54 & 1.28 & 1.02 & $3.28 * * *$ & 1.37 \\
\hline INC-CON after inc & $t(19)$ & $4.42 * * *$ & 1.48 & $<1$ & $<1$ & 1.18 & $<1$ & $<1$ & $<1$ \\
\hline INC-CON after con vs. inc & $t(19), 1-\mathrm{t}$ & $3.62 * * *$ & $2.49 * *$ & $3.80 * * *$ & $2.39 * *$ & $3.65 * * *$ & $3.98 * * *$ & $3.20 * * *$ & $3.45 * * *$ \\
\hline INC-CON after con vs. non & $t(19), 1-\mathrm{t}$ & $2.54 * *$ & $2.76 * *$ & $2.65 * *$ & $1.82 * *$ & $2.39 * *$ & $3.03 * * *$ & 1.04 & $3.00 * * *$ \\
\hline INC-CON after non vs. inc & $t(19), 1-\mathrm{t}$ & $2.49 * *$ & $1.49 *$ & $<1$ & $<1$ & $1.79 * *$ & 1.08 & $2.32 * *$ & $<1$ \\
\hline \multicolumn{10}{|l|}{ Valid Cueing } \\
\hline conCON vs. nonCON & $t(19), 1-\mathrm{t}$ & $2.43 * *$ & $<1$ & $<1$ & $<1$ & $<1$ & $<1$ & 1.09 & $<1$ \\
\hline incINC vs. nonINC & $t(19), 1-\mathrm{t}$ & $1.68 *$ & 1.16 & $<1$ & $<1$ & $<1$ & $<1$ & $<1$ & $<1$ \\
\hline \multicolumn{10}{|l|}{ Invalid Cueing } \\
\hline conINC vs. nonINC & $t(19), 1-\mathrm{t}$ & $2.06 * *$ & $2.86 * * *$ & $2.57 * *$ & $2.14 * *$ & $2.78 * *$ & $2.51 * *$ & $2.43 * *$ & $2.37 * *$ \\
\hline incCON vs. nonCON & $t(19), 1-\mathrm{t}$ & $1.79 * *$ & $2.04 * *$ & $<1$ & $<1$ & $2.81 * *$ & 1.02 & $3.37 * * *$ & $<1$ \\
\hline
\end{tabular}

$\mathrm{df}=$ degrees of freedom; $\mathrm{RT}=$ reaction time; $\mathrm{ERR}=$ error rate; $\mathrm{ACC}=$ anterior cingulate cortex; DLPFC $=$ dorsolateral prefrontal cortex $(\mathrm{l}=\mathrm{left} ; \mathrm{r}=$ right); INC = incongruent targets; $\mathrm{CON}=$ congruent targets; $\mathrm{con}=$ congruent-predicting cues; non $=$ nonpredictive cues; inc $=$ incongruent-predicting cues; 1 - $\mathrm{t}=$ one-tailed.

${ }^{\mathrm{a}}$ ROIs selected on the basis of the Stroop conflict contrast following congruent-predicting cues $\left(p_{\mathrm{FDR}}<.05\right)$.

${ }^{\mathrm{b}}$ ROIs selected on the basis of the Stroop conflict contrast following all cue types ( $p_{\text {uncorr }}<.005$ ).

${ }^{*} p<.1$ (trend).

$* * p<.05$.

$* * * p<.005$

conflict effect after congruent-predicting than nonpredictive cues. Thus, also in error rates, the conflict effect was larger following congruent-predicting cues than incongruent-predicting and nonpredictive cues (Figure 2B and Table 1). The error rates did not reveal effects of valid cueing. However, as with the RTs, there was an effect of invalid cueing: Incongruent targets induced more errors when preceded by invalid congruent-predicting cues than when preceded by nonpredictive cues.

In summary, the RTs and error rates were differentially affected by cue condition, providing evidence for cuebased adjustment of attentional control. Performance benefited from valid cueing (especially for congruent targets) and was hampered by invalid cueing.

\section{Neuroimaging Results}

Conflict effects in the brain were dependent on the preceding predictive or nonpredictive cues. A conflict effect was observed following congruent-predicting cues only, among others, in two adjacent regions in dorsal ACC (dACC), with a peak in ACC and a peak in the pre-SMA (Figure 3A). Even after lowering the statistical threshold, no ACC activity was observed for incongruent versus congruent targets following incongruent-predicting cues or following nonpredictive cues. Regions showing activation and deactivation for the conflict contrast after congruentpredicting cues are listed in Table 2. Statistics of targetrelated imaging results are mentioned in Table 1.

\section{Target-related Anterior Cingulate Cortex Activity}

To demonstrate dACC responses to the targets, subjectaveraged beta-weights were extracted for all voxels in the dACC region showing increased activity for incongruent compared with congruent targets following congruentpredicting cues (Figure 3A and B, left). As indicated, there was only a conflict effect after congruent-predicting 
cues. As with the behavioral results, dACC activity was enhanced when incongruent targets were invalidly cued by congruent-predicting cues compared with nonpredictive cues. However, a similar effect of invalid cueing was not observed for congruent targets following incongruentpredicting cues.

To investigate whether similar target-related results would be obtained if ACC ROI is selected with a more general contrast, we extracted beta-weights from the ACC region that was activated for incongruent relative to congruent targets following all three cue types (i.e., without introducing a selection bias towards conflict effects following a certain cue type) (Figure 4A and B, left). This was done at an uncorrected threshold $(p<.005)$ as no whole-brain activity was obtained for this contrast at a corrected threshold. Nevertheless, the ACC region that was activated by targets irrespective of preceding cue type (BA 32; peak Talairach coordinates: $x=6, y=32, z=31$; cluster size $=196 \mathrm{~mm}^{3}$ ) showed similar target-related effects as did the dACC region activated by targets following congruent-predicting cues (Figures 3B and 4B, left; Table 1). In the ROI that was activated by targets regardless of cue condition, a conflict effect occurred only after congruent-predicting cues. Furthermore, ACC activity was enhanced when incongruent targets were invalidly cued by congruent-predicting compared with nonpredictive cues. Again, invalid cueing yielded no such effect for congruent targets.

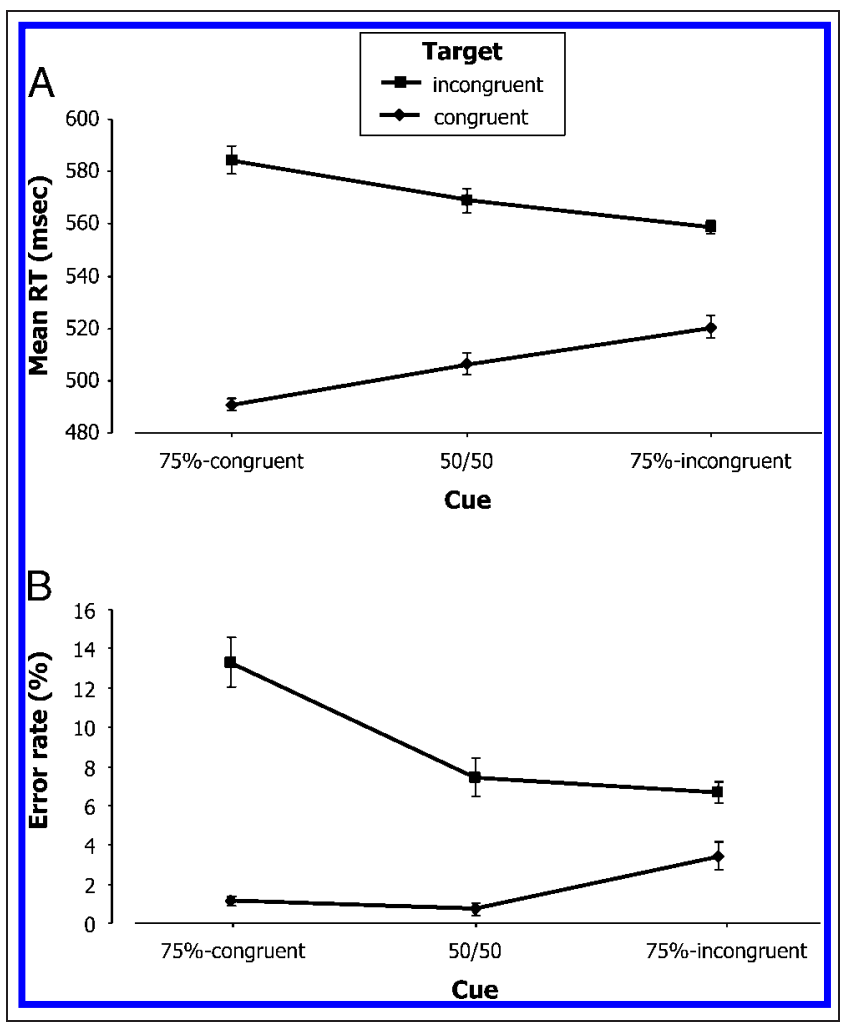

Figure 2. Behavioral results. (A) Mean RTs and (B) error rates of congruent and incongruent target condition preceded by predictive and nonpredictive cues. Error bar is standard error of mean.
In summary, the cues influenced reactive, target-related control in ACC. In both the whole-brain and ROI analyses of target-related activity, a conflict effect in ACC was observed only when targets were preceded by congruent-predicting cues (Figure 3A and B, Figure 4A and B, left; Table 1). Analysis of the preceding cue-related activity in ACC is reported below.

In the whole-brain analyses, we found no differential activity among the three cue types (congruent-predicting, incongruent-predicting, nonpredictive). To investigate the activity pattern evoked by the preceding cues in the conflict-related areas, we looked for cue-related effects in the ROIs determined by the target-related contrast (see above). Furthermore, we looked at cue-evoked activity in ROIs from our previous study that used similar cues in a comparable design (Aarts et al., 2008), and see Methods section.

\section{Cue-related Anterior Cingulate Cortex Activity}

From the dACC ROI that showed increased activity for incongruent compared with congruent targets following congruent-predicting cues, cue-related subject-averaged beta-weights were extracted and AACC responses to cues were tested with paired-sample $t$ tests (Figure 3A and C, left). Congruent-predicting cues elicited marginally more activity in dACC than nonpredictive cues $[t(19)=1.84$, $p=.082$, two-tailed]. Incongruent-predicting cues did not elicit more activity than nonpredictive cues $[t(19)<$ $1]$ or congruent-predicting cues $[t(19)=1.67, p=.11$, two-tailed].

The ACC region that was activated in Aarts et al. (2008) for $100 \%$ predictive cues versus nonpredictive cues (Figure $5 \mathrm{~A}$, left) showed no differential cue-related activity in the present experiment [congruent-predicting vs. nonpredictive cues: $t(19)=1.23, p=.24$, two-tailed; congruentpredicting vs. incongruent-predicting cues: $t(19)<1$; incongruent-predicting vs. nonpredictive cues: $t(19)=$ $1.55, p=.14$, two-tailed] (Figure 5B, left).

In summary, the ACC region that showed a Stroop effect at targets following congruent-predicting cues did not show differential activity for the different cue types (Figure $3 \mathrm{~A}$ and $\mathrm{C}$, left). In an unbiased ACC ROI from a previous study (Aarts et al., 2008), there was no differential cuerelated activity either (Figure 5A and B, left).

\section{Target-related Dorsolateral Prefrontal Cortex Activity}

Similar ROI analyses were performed on the target-related subject-averaged beta-weights of left DLPFC (BA 9, Talairach coordinates: $x=-54, y=14, z=28$ ) and right DLPFC (BA 8/9, Talairach coordinates: $x=45, y=11, z=40$ ) that showed increased activity for incongruent compared with congruent targets following congruent-predicting cues (see Figure 3A and B, right; Table 1). For illustrative purposes, we mention the DLPFC responses to the targets. DLPFC showed a pattern of activity similar to that observed 
Figure 3. dACC activity and DLPFC activity. (A) Group maps (sagittal section) showing increased activity in (left) adjacent regions in medial frontal cortex: ACC (peak Talairach coordinates: $x=6$, $y=32, z=31$ ) and pre-SMA (peak Talairach coordinates: $x=6, y=14, z=49)$, and (right) left DLPFC (peak Talairach coordinates: $x=-54$, $y=14, z=28$ ) for incongruent compared with congruent targets following congruentpredicting cues (thresholded at $p<.05$, false discovery rate corrected). (B) Mean beta-weights for the targets and (C) mean beta-weights for the cues in the dACC region (left) and the DLPFC region (right) that showed an effect of incongruent targets versus congruent targets following congruent-predicting cues. Error bar is standard error of mean.

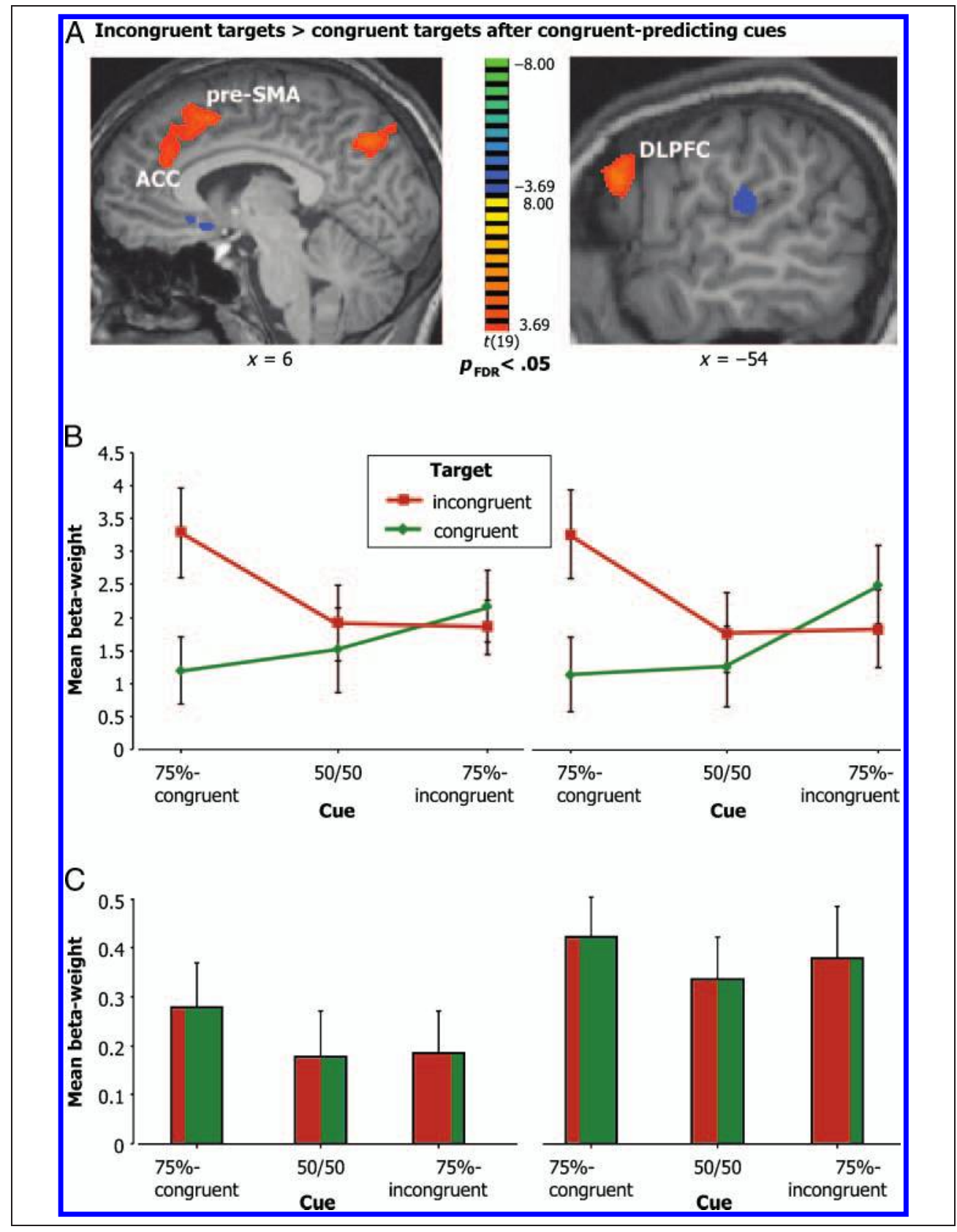

in dACC. There was only a conflict effect after congruentpredicting cues. DLPFC activity was enhanced when incongruent targets were invalidly cued by congruent-predicting cues compared with nonpredictive cues, and a similar effect of invalid cueing was observed for congruent targets in left DLPFC, but not in right DLPFC.

To investigate target-related DLPFC activity in the ROIs selected on the basis of a more general contrast (see Targetrelated Anterior Cingulate Cortex Activity above), we extracted beta-weights from the DLPFC regions that were activated for incongruent relative to congruent targets following all three cue types at $p<.005$ (left DLPFC, BA 9; peak Talairach coordinates: $x=-51, y=11, z=37$; cluster size $=116 \mathrm{~mm}^{3}$, Figure $4 \mathrm{~A}$ and $\mathrm{B}$, right; right DLPFC, BA 9; peak Talairach coordinates: $x=39, y=11, z=37$; cluster size $=864 \mathrm{~mm}^{3}$ ). In the left DLPFC ROI, there were conflict effects after both congruent-predicting and nonpredictive cues, which did not differ significantly from each other (see Table 1). DLPFC activity was enhanced when incongruent targets were invalidly cued by congruentpredicting cues compared with nonpredictive cues, and a similar effect of invalid cueing was observed for congruent targets in left DLPFC, but not in right DLPFC.

Thus, conflict effects in DLPFC were more evident following congruent-predicting cues than following the other cues (Figure 3A and B, right; Table 1), similar to what was observed for ACC. However, when the ROIs were selected on the basis of a more general Stroop conflict contrast, left DLPFC also showed a conflict effect following nonpredictive cues (Figure 4A and B, right; Table 1). 
Cue-related Dorsolateral Prefrontal Cortex Activity

From the DLPFC ROIs that showed increased activity for incongruent compared with congruent targets following congruent-predicting cues, cue-related subject-averaged beta-weights were extracted and DLPFC responses to cues were tested with paired-sample $t$ tests (Figure 3A and $\mathrm{C}$, right). Incongruent-predicting cues did not elicit more activity in DLPFC than nonpredictive cues [left + right: $t(19)<1]$ or congruent-predicting cues [left +

Table 2. Peak Talairach Coordinates, Peak $t$ Values, and Cluster Size $\left(\mathrm{mm}^{3}\right)$ of Regions Showing a Conflict Effect (Incongruent $>$ Congruent Trials) following Congruent-predicting Cues for $p<.05$ (False Discovery Rate Corrected) and a Threshold of $50 \mathrm{~mm}^{3}$

\begin{tabular}{|c|c|c|c|c|c|c|}
\hline Region & $B A^{a}$ & $x$ & $y$ & $z$ & $t(19)$ & Size \\
\hline \multicolumn{7}{|l|}{ Activations } \\
\hline Presupplementary motor area, right & 6 & 6 & 14 & 49 & 5.17 & 2715 \\
\hline Anterior cingulate cortex, right & 32 & 6 & 32 & 31 & 4.55 & \\
\hline Middle frontal gyrus, left & 10 & -36 & 62 & 7 & 4.73 & 450 \\
\hline Inferior frontal gyrus, right & 10 & 45 & 53 & 1 & 5.51 & 3899 \\
\hline Inferior frontal gyrus, left & 47 & -30 & 20 & 1 & 4.73 & 234 \\
\hline Inferior frontal gyrus, right & 47 & 33 & 20 & -2 & 5.52 & 1564 \\
\hline Dorsolateral prefrontal cortex, left & 9 & -54 & 14 & 28 & 5.59 & 2247 \\
\hline Dorsolateral prefrontal cortex, right & $8 / 9$ & 45 & 11 & 40 & 6.64 & 9592 \\
\hline Precentral gyrus, left & 6 & -30 & -10 & 52 & 4.09 & 57 \\
\hline Superior temporal gyrus, right & 38 & 51 & 14 & -26 & 5.09 & 561 \\
\hline Middle temporal gyrus, right & 21 & 54 & -28 & -5 & 5.26 & 1781 \\
\hline Inferior temporal gyrus, right & 37 & 57 & -49 & -20 & 4.62 & 99 \\
\hline Posterior cingulate gyrus, right & 23 & 3 & -25 & 31 & 4.32 & 71 \\
\hline Precuneus, right & 7 & 3 & -67 & 37 & 6.09 & 2230 \\
\hline Inferior parietal lobe, right & 40 & 33 & -52 & 37 & 10.59 & 13859 \\
\hline Inferior parietal lobe, left & 40 & -30 & -52 & 37 & 7.30 & 8200 \\
\hline Cerebellum, left & & -30 & -55 & -35 & 6.01 & 3590 \\
\hline Cerebellum, right & & 30 & -55 & -32 & 4.59 & 143 \\
\hline \multicolumn{7}{|l|}{ Deactivations } \\
\hline Middle frontal gyrus, left & 11 & -18 & 29 & 1 & -4.42 & 69 \\
\hline Subgenual area, right & 25 & 6 & 23 & -2 & -4.53 & 369 \\
\hline Cingulate gyrus, left & 24 & -3 & 2 & 28 & -4.07 & 70 \\
\hline Superior frontal gyrus, right & 6 & 18 & -7 & 67 & -4.99 & 66 \\
\hline Insula, left & 13 & -42 & -4 & 1 & -4.34 & 129 \\
\hline Insula, left & 13 & -42 & -13 & 13 & -4.39 & 249 \\
\hline Insula, right & 13 & 36 & -13 & 22 & -5.05 & 1775 \\
\hline Posterior cingulate gyrus, left & 30 & -15 & -52 & 16 & -4.79 & 422 \\
\hline Posterior cingulate gyrus, right & 31 & 15 & -34 & 40 & -4.88 & 406 \\
\hline Inferior parietal lobe, left & 40 & -57 & -28 & 19 & -4.35 & 606 \\
\hline Parahippocampal/fusiform gyrus, right & 37 & 33 & -40 & -8 & -5.44 & 617 \\
\hline Parahippocampal/fusiform gyrus, left & 37 & -33 & -40 & -11 & -5.47 & 102 \\
\hline
\end{tabular}

${ }^{\mathrm{a}} \mathrm{BA}=$ Brodmann's area 
Figure 4. Target-related activity in ACC and DLPFC. (A) Group maps (sagittal section) showing increased activity in (left) ACC (peak Talairach coordinates: $x=6$, $y=32, z=31$; cluster size $=$ $196 \mathrm{~mm}^{3}$ ), and (right) left DLPFC (peak Talairach coordinates: $x=-51, y=11$, $z=37$; cluster size $=116 \mathrm{~mm}^{3}$ ) for incongruent compared with congruent targets following all cue types (thresholded at $p<.005$, uncorrected for multiple comparisons). (B) Mean beta-weights for the and the DLPFC region (right) that showed an effect of incongruent targets versus congruent targets following all cue types. Error bar is standard error of mean. targets in ACC region (left)

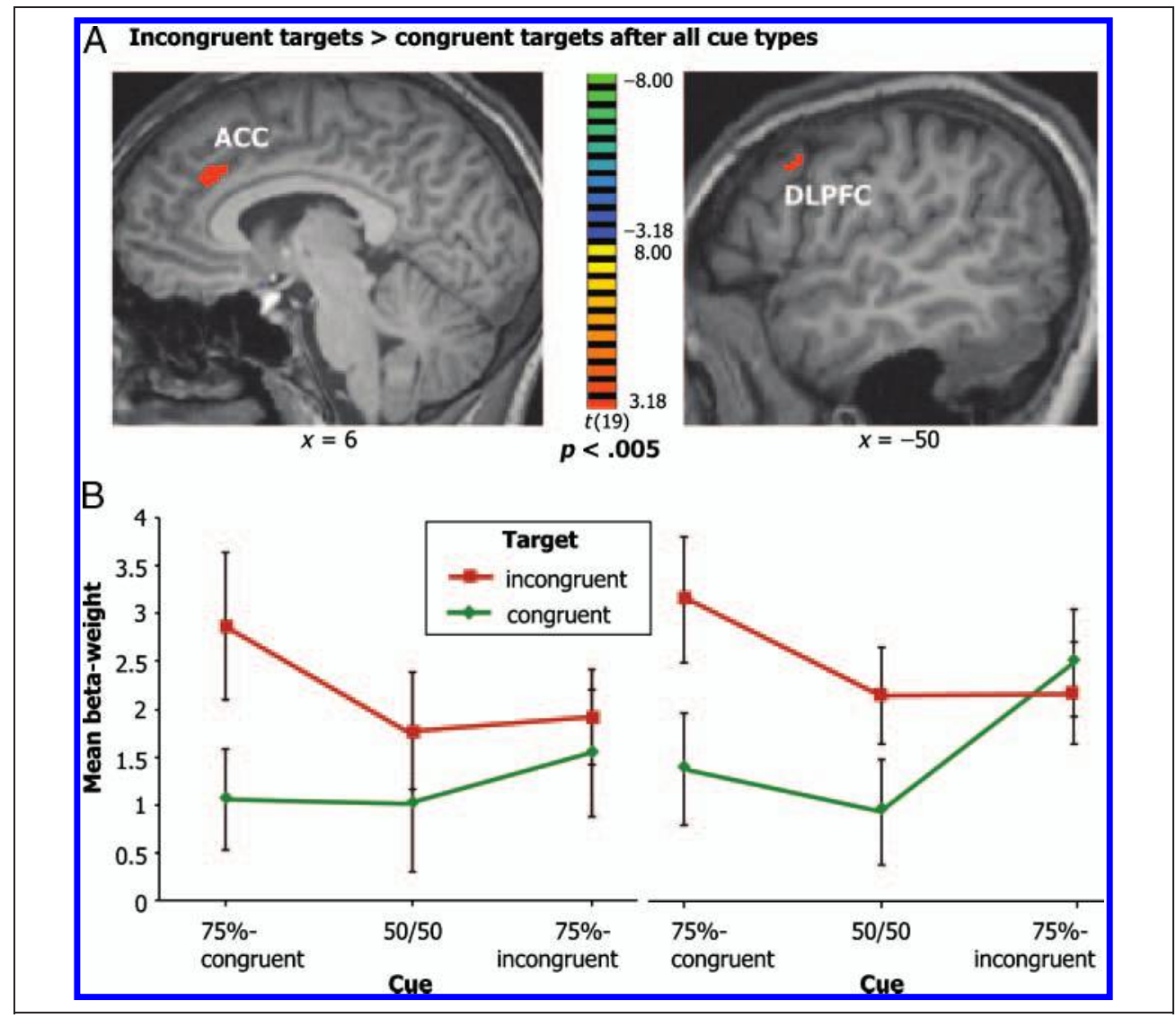

Figure 5. Cue-related activity in ACC and DLPFC ROIs from Aarts et al. (2008). (A) Sagittal sections showing ACC (left) and left DLPFC (right) ROIs from Aarts et al. showing an effect of $100 \%$ predictive versus nonpredictive cues (ACC: $x=-9, y=14$, $z=37$, cluster size $=$ $1762 \mathrm{~mm}^{3}$; DLPFC: $x=-27$, $y=26, z=28$, cluster size $=316 \mathrm{~mm}^{3}$ ). (B) Mean beta-weights for the cues in ACC ROI (left) and the DLPFC ROI (right) from Aarts et al. Error bar is standard error of mean.

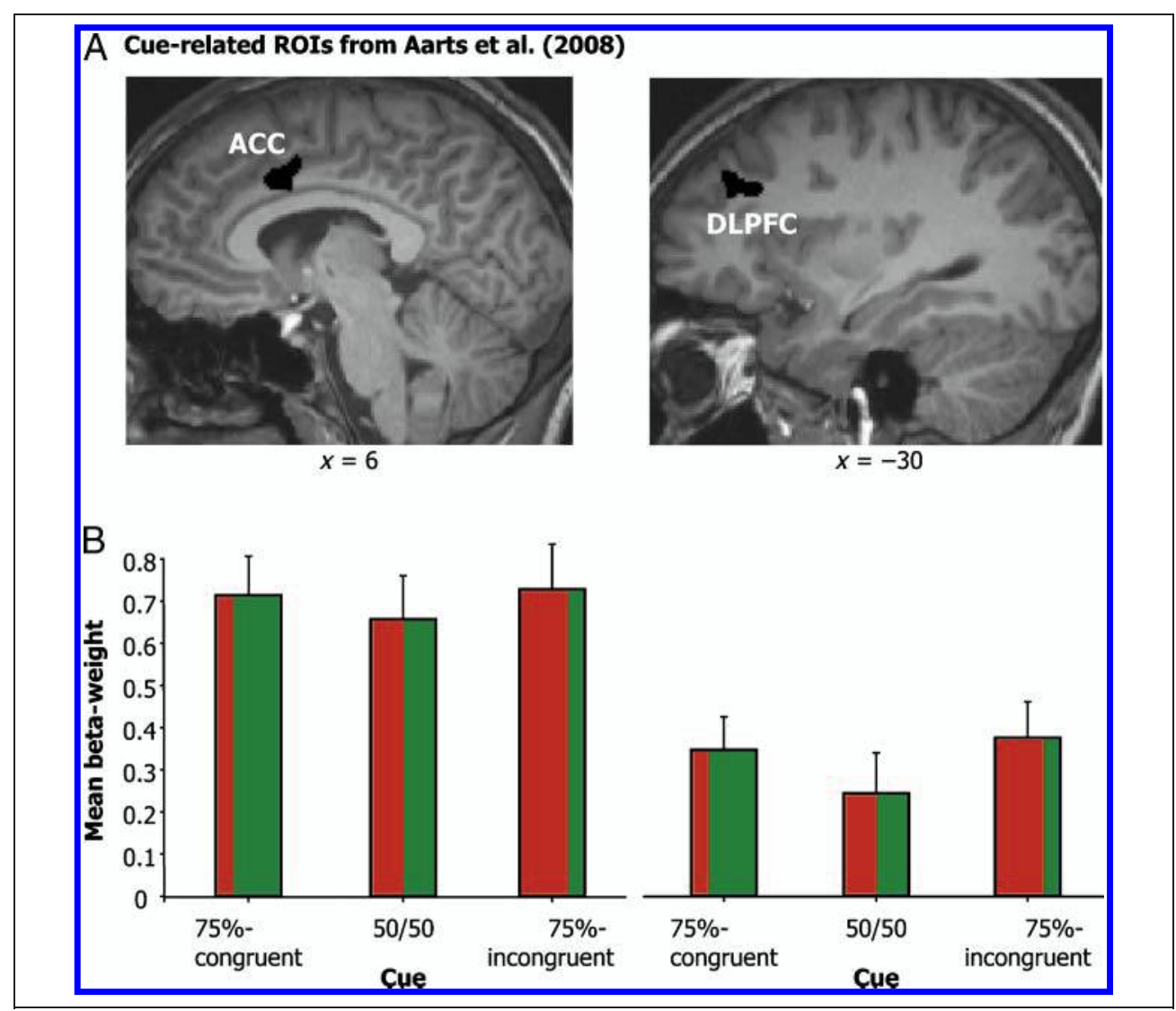


right: $t(19)<1$ ] . Congruent-predicting cues also did not elicit more activity in DLPFC than did nonpredictive cues [left: $t(19)=1.56, p=.13$, two-tailed; right: $t(19)=1.71$, $p=.10$, two-tailed].

Similar to the target-related ACC region, the left DLPFC region that was activated in Aarts et al. (2008) for 100\% predictive cues versus nonpredictive cues (Figure 5A, right) showed marginally more activity for congruent-predicting cues than for nonpredictive cues $[t(19)=1.91, p=.07$, two-tailed] (Figure 5B, right). Moreover, this ROI in left DLPFC demonstrated more activity for incongruentpredicting cues than for nonpredictive cues $[t(19)=2.50$, $p=.022$, two-tailed] (Figure 5B, right). However, these differences were not significant when corrected for multiple comparisons. Activity in left DLPFC did not differ between congruent-predicting and incongruent-predicting cues $[t(19)<1]$. In the ROI in right DLPFC, there were no differential cue-related effects [all $t(19)<1$ ].

To summarize, in the DLPFC regions that only showed a conflict effect following congruent-predicting cues, there were no differential effects for the different cue types preceding the Stroop targets (Figure $3 \mathrm{~A}$ and $\mathrm{C}$, right). In the unbiased DLPFC ROIs from our previous study (Aarts et al., 2008), only left DLPFC showed significant cue-related effects (Figure $5 \mathrm{~A}$ and $\mathrm{B}$, right). However, these differences were not significant when correcting for multiple comparisons.

\section{DISCUSSION}

In the present fMRI study, we tested whether Stroop-like conflict activity in ACC is influenced by preceding cues in a similar fashion as has been reported for the level of conflict on a preceding trial (Kerns et al., 2004; Botvinick et al., 1999). To this end, we specifically manipulated participants' expectancies by using symbolic cues that predicted the upcoming Stroop-like target condition with $75 \%$ or $50 \%$ certainty. Similar to the effects observed when looking at Stroop conflict as a function of trial-to-trial sequences (Kerns et al., 2004; Botvinick et al., 1999), we observed increased conflict effects following congruent-predicting cues compared with incongruent-predicting cues both behaviorally and in target-related dACC activity. Furthermore, comparable to previous studies investigating Stroop conflict following incongruent trials (e.g., Botvinick et al., 1999), there was no conflict effect following incongruentpredicting cues in ACC, despite a behavioral conflict effect. In contrast, performance costs after invalid congruentpredicting cues (on incongruent targets) resulted in an increased Stroop conflict effect observed in ACC and behavior following these cues. Thus, the present data show that enhanced Stroop conflict effects in ACC can be the result of specific control adjustments evoked by symbolic cues.

The present findings suggest that adjustment of attentional control may be expectancy-based rather than only conflict-driven. Although behavioral and dACC effects replicate those of previous studies taken as evidence of control adjustments on the basis of experienced conflict (Kerns et al., 2004; Botvinick et al., 1999), our data demonstrate that these results can also be obtained by presenting cues signaling the probability of upcoming congruent or incongruent targets instead of the actual occurrence of conflict. Thus, the present results suggest that cues that explicitly induce expectations about the upcoming Stroop target condition can just as well evoke control adjustments (cf. Bartholow et al., 2005; Gratton et al., 1992), which, in turn, can lead to altered Stroop conflict effects in ACC. We suggest that participants engage in a strategy of widening of attention when expecting a congruent trial (allowing more influence of the distractor) and a focusing of attention when expecting an incongruent trial (reducing the influence of the distractor). Hence, when expectancies are confirmed, performance benefits from these proactive adjustments, as was observed when cues were valid in the present experiment. In contrast, when expectancies are violated, reactive control has to be implemented because proactive control was inappropriate, resulting in a performance cost.

RTs, error rates, and dACC activity were most prominently increased when an incongruent target was invalidly cued, thus, when a congruent target was expected. In terms of strategic adjustments, an invalidly cued incongruent target is more costly for performance than an invalidly cued congruent target. This is because the widening of attention increases RTs and errors for incongruent targets, whereas the focusing of attention is only a suboptimal strategy for congruent targets. Hence, when an incongruent target is invalidly cued, the need to adjust control is most critical and activity of dACC, implementing the reactive, targetbased control, is highest (Roelofs et al., 2006). This contrasts with the lack of differential reactive control following predictive cues in our previous study (Aarts et al., 2008), which is explained by the fact that the cues were always valid in that study, thus, expectancies were never violated. However, expectancy violation alone cannot explain the present target-related results in ACC because the effects of expectancy violation should be equal for congruent and incongruent targets, whereas ACC only showed effects of invalid cueing for incongruent targets. Activity in left DLPFC did show effects of invalid cueing for both congruent and incongruent targets, similar to what was observed in the behavioral results. It is unclear whether DLPFC is more sensitive than ACC to the less optimal strategy of focusing of attention for processing congruent targets, or whether DLPFC is more sensitive to expectancy violation than ACC. Whether the effects in DLPFC are wholly or partly due to expectancy violation is an issue for future research.

In a previous study, we showed that ACC activity was equally enhanced for 100\%-valid congruent-predicting and incongruent-predicting cues compared with nonpredictive cues (Aarts et al., 2008). Therefore, we suggested that ACC is involved in making strategic adjustments in control independent of the specific processing strategies (i.e., widening or focusing of attention). However, in the present 
study using probabilistic cues, we did not observe any differential cue-related activity in the whole-brain or ROI analysis. This suggests that, although the reactive control effects in dACC are dependent on proactive control during the cues, the activity in dACC during proactive control itself is less clear than observed previously with 100\%valid cues. Although the focus of the present study was on Stroop conflict effects as a function of preceding cues, the question remains why cue-related effects were less pronounced in the present study than in the previous study (Aarts et al., 2008). We hypothesize that the amount of proactive control elicited by the cues is dependent on the validity of these cues. In our previous study, activity for the predictive cues clearly differed from that of the nonpredictive cues. This is probably due to the fact that the predictive cues predicted an upcoming target with $100 \%$ probability and the nonpredictive cues predicted an upcoming target with 33\% probability. In the present study, the difference in probability signaled by the cues was only $25 \%$ ( $75 \%$ predictive cues vs. $50 \%$ nonpredictive cues) instead of $67 \%$. Hence, the difference in proactive control activity in ACC elicited by the different cues is presently less evident because all three cue types elicited expectations, and thus, most likely all triggered control adjustments. Nonetheless, the cue manipulation in the present study was clearly sufficient to induce behavioral and neural changes in reactive control during processing of the Stroop targets. This indicates that proactive control in response to the cues indeed took place, although the difference in brain activity between the cues is less apparent than in our previous study (Aarts et al., 2008).

Regarding proactive and reactive control, one can make an analogy with task switching. In task switching, cuerelated proactive control can influence target-related reactive control, that is, by reducing switch costs (Monsell, Sumner, \& Waters, 2003). Yet, no matter how much preparation is allowed, a switch cost remains (De Jong, 2000; Rogers \& Monsell, 1995). This is comparable with the behavioral Stroop conflict effect that can still be observed despite cues predicting the upcoming congruency with $100 \%$ validity (Aarts et al., 2008). When Stroop-like interference was investigated in a task-switching setting, it was shown that task cues could reduce the switch cost but not the interference on the targets (Monsell et al., 2003). Hence, it was concluded that control of interference is purely reactive, and control is only evoked after detection of conflict. However, the present and previous results (Aarts et al., 2008) show that reactive control of interference can also be influenced by symbolic cues instead of conflict. In our experiments, this proactive control is evoked by cues predicting upcoming congruency instead of upcoming tasks. Proactive control in ACC has also been observed with task cues (e.g., Luks, Simpson, Feiwell, \& Miller, 2002). Moreover, we have recently demonstrated that ACC is engaged by both response and task conflict elicited in a task-switching Stroop task (Aarts, Roelofs, \& van Turennout, 2009).
The present experiment provides evidence of the involvement of ACC in expectancy-based adjustments in attentional control evoked by probabilistic cues. However, we do not want to claim that subjects' expectancies are solely responsible for control adjustments. It is possible that conflict-driven and episodic memory factors (Hommel, Proctor, \& Vu, 2004; Mayr, Awh, \& Laurey, 2003) contribute to these adjustments, too. These latter reactive control effects might exist besides the proactive control effects we observed in the present study. To what extent these different types of control adjustments play a role may differ among task situations and contexts (Fernandez-Duque \& Knight, 2008). A cued paradigm with $75 \%$-valid cues need not be fully comparable with a trial-to-trial experimental paradigm. However, most importantly, our present and previous data (Aarts et al., 2008) do show that the actual occurrence of conflict is not required to evoke strategic adjustments, but that such adjustments can also be generated by cues signaling the probability of congruent or incongruent trials. Therefore, expectancy-based control adjustments should be taken into account when studying sequential effects and the role of ACC.

\section{Acknowledgments}

We thank Roshan Cools for helpful comments on an earlier version of this manuscript. This work was supported by a VICI grant of the Netherlands Organisation for Scientific Research to A. R.

Reprint requests should be sent to Esther Aarts, Donders Centre for Cognitive Neuroimaging, P.O. Box 9101, 6500 HB Nijmegen, The Netherlands, or via e-mail: e.aarts@donders.ru.nl.

\section{REFERENCES}

Aarts, E., Roelofs, A., \& van Turennout, M. (2008). Anticipatory activity in anterior cingulate cortex can be independent of conflict and error likelihood. Journal of Neuroscience. 28, 4671-4678.

Aarts, E., Roelofs, A., \& van Turennout, M. (2009). Attentional control of task and response in lateral and medial frontal cortex: Brain activity and reaction time distributions. Neuropsychologia, 47, 2089-2099.

Baldo, J. V., Shimamura, A. P., \& Prinzmetal, W. (1998). Mapping symbols to response modalities: Interference effects on Stroop-like tasks. Perception \& Psychophysics, 60, 427-437.

Bartholow, B. D., Pearson, M. A., Dickter, C. L., Sher, K. J., Fabiani, M., \& Gratton, G. (2005). Strategic control and medial frontal negativity: Beyond errors and response conflict. Psychopbvsiology 42, 33-42.

Botvinick, M. M., Braver, T. S., Barch, D. M., Carter, C. S., \& Cohen, J. D. (2001). Conflict monitoring and cognitive control. Psvchological Review, 108, 624-652.

Botvinick, M. M., Cohen, J. D., \& Carter, C. S. (2004). Conflict monitoring and anterior cingulate cortex: An update. Trends in Cognitive Sciences, 8, 539-546.

Botvinick, M. M., Nystrom, L. E., Fissell, K., Carter, C. S., \& Cohen, J. D. (1999). Conflict monitoring versus selectionfor-action in anterior cingulate cortex. Nature, 402, 179-181.

Bush, G., Luu, P., \& Posner, M. I. (2000). Cognitive and emotional influences in anterior cingulate cortex. Trends in Cognitive Sciences, 4, 215-222. 
De Jong, R. (2000). An intention-activation account of residual switch costs. In S. Monsell \& J. S. Driver (Eds.), Control of cognitive processes: Attention and performance XVIII (pp. 357-376). Cambridge, MA: MIT Press.

De Pisapia, N., \& Braver, T. S. (2006). A model of dual control mechanisms through anterior cingulate and prefrontal cortex interactions. Neurocomputing, 69, 1322-1326.

Duncan, J. (1995). Attention, intelligence, and the frontal lobes. In M. Gazzaniga (Ed.), The cognitive neurosciences (pp. 721-733). Cambridge, MA: MIT Press.

Duncan, J. (2005). Frontal lobe function and general intelligence: Why it matters. Cortex, 41, 215-217.

Egner, T. (2007). Congruency sequence effects and cognitive control. Cognitive. Affective \& Behavioral Neuroscience, 7, 380-390.

Fernandez-Duque, D., \& Knight, M. (2008). Cognitive control: Dynamic, sustained, and voluntary influences. Journal of Experimental Psychology: Human Perception and Performance, 34, 340-355.

Genovese, C. R., Lazar, N. A., \& Nichols, T. (2002). Thresholding of statistical maps in functional neuroimaging using the false discovery rate. Neuroimage, 15, 870-878.

Gratton, G., Coles, M. G., \& Donchin, E. (1992). Optimizing the use of information: Strategic control of activation of responses. Journal of Experimental Psychology: General, 121, 480-506.

Hommel, B., Proctor, R. W., \& Vu, K. P. (2004). A featureintegration account of sequential effects in the Simon task. Psychological Research, 68, 1-17.

Josephs, O., Turner, R., \& Friston, K. (1997). Event-related fMRI. Human Brain Mapping, 5, 243-248.

Kerns, J. G., Cohen, J. D., MacDonald, A. W., III, Cho, R. Y., Stenger, V. A., \& Carter, C. S. (2004). Anterior cingulate conflict monitoring and adjustments in control. Science. 303, 1023-1026.

Logan, G. D., \& Zbrodoff, N. J. (1979). When it helps to be misled: Facilitative effects of increasing the frequency of conflicting stimuli in a Stroop-like task. Memorv $\mathcal{E}$ Cognition, 7, 166-174.

Logan, G. D., \& Zbrodoff, N. J. (1982). Constraints on strategy construction in a speeded discrimination task. Journal of Experimental Psychology: Human Perception and Performance, 8, 502-520.

Luks, T. L., Simpson, G. V., Feiwell, R. J., \& Miller, W. L. (2002). Evidence for anterior cingulate cortex involvement in monitoring preparatory attentional set. Neuroimage, 17 , 792-802.

MacLeod, C. M. (1991). Half a century of research on the Stroop effect: An integrative review. Psvchological Bulletin. 109, 163-203.

Mayr, U., Awh, E., \& Laurey, P. (2003). Conflict adaptation effects in the absence of executive control. Nature Neuroscience, 6, 450-452.

Miller, E. K. (2000). The prefrontal cortex and cognitive control. Nature Reviews Neuroscience, 1, 59-65.

Monsell, S., Sumner, P., \& Waters, H. (2003). Task-set reconfiguration with predictable and unpredictable task switches. Memory \& Cognition, 31, 327-342.

Orr, J. M., \& Weissman, D. H. (2009). Anterior cingulate cortex makes 2 contributions to minimizing distraction. Cerebral Cortex, 19, 703-711.

Paus, T. (2001). Primate anterior cingulate cortex: Where motor control, drive and cognition interface. Nature Reviews Neuroscience, 2, 417-424.

Picard, N., \& Strick, P. L. (1996). Motor areas of the medial wall: A review of their location and functional activation. Cerebral Cortex, 6, 342-353.

Roelofs, A., van Turennout, M., \& Coles, M. G. (2006). Anterior cingulate cortex activity can be independent of response conflict in Stroop-like tasks. Proceedings of the National Academy of Sciences, U.S.A., 103, 13884-13889.

Rogers, R. D., \& Monsell, S. (1995). Costs of a predictable switch between simple cognitive tasks. Journal of Experimental Psychology: General, 124, 207-231.

Stroop, J. R. (1935). Studies of interference in serial verbal reactions. Journal of Experimental Psychology, 18, 643-662.

Talairach, J., \& Tournoux, P. (1988). Co-planar stereotaxic atlas of the buman brain. Stuttgart, Germany: Thieme.

Turken, A. U., \& Swick, D. (1999). Response selection in the human anterior cingulate cortex. Nature Neuroscience, 2, 920-924.

Weissman, D. H., Gopalakrishnan, A., Hazlett, C. J., \& Woldorff, M. G. (2005). Dorsal anterior cingulate cortex resolves conflict from distracting stimuli by boosting attention toward relevant events. Cerebral Cortex, 15, 229-237.

Wuhr, P., \& Kunde, W. (2008). Precueing spatial S-R correspondence: Is there regulation of expected response conflict? Journal of Experimental Psychology: Human Perception and Performance, 34, 872-883. 


\section{This article has been cited by:}

1. Xiao Lin, Guangheng Dong, Qiandong Wang, Xiaoxia Du. 2015. Abnormal gray matter and white matter volume in 'Internet gaming addicts'. Addictive Behaviors 40, 137-143. [CrossRef]

2. Tobias Egner. 2014. Creatures of habit (and control): a multi-level learning perspective on the modulation of congruency effects. Frontiers in Psychology 5. . [CrossRef]

3. R. Gaschler, S. Schwager, V.J. Umbach, P.A. Frensch, T. Schubert. 2014. Expectation mismatch: Differences between selfgenerated and cue-induced expectations. Neuroscience \& Biobehavioral Reviews 46, 139-157. [CrossRef]

4. Wout Duthoo, Elger L. Abrahamse, Senne Braem, Carsten N. Boehler, Wim Notebaert. 2014. The heterogeneous world of congruency sequence effects: an update. Frontiers in Psychology 5. . [CrossRef]

5. William H. Alexander, Joshua W. Brown. 2014. A general role for medial prefrontal cortex in event prediction. Frontiers in Computational Neuroscience 8. . [CrossRef]

6. W. Duthoo, E. L. Abrahamse, S. Braem, W. Notebaert. 2014. Going, going, gone? Proactive control prevents the congruency sequence effect from rapid decay. Psychological Research 78, 483-493. [CrossRef]

7. Taolin Chen, Keith Maurice Kendrick, Chunliang Feng, Suyong Yang, Xiaogang Wang, Xun Yang, Du Lei, Min Wu, Xiaoqi Huang, Qiyong Gong, Yuejia Luo. 2014. Opposite effect of conflict context modulation on neural mechanisms of cognitive and affective control. Psychophysiology 51:10.1111/psyp.2014.51.issue-5, 478-488. [CrossRef]

8. Samuel Nastase, Vittorio Iacovella, Uri Hasson. 2014. Uncertainty in visual and auditory series is coded by modality-general and modality-specific neural systems. Human Brain Mapping 35:10.1002/hbm.v35.4, 1111-1128. [CrossRef]

9. Chunming Luo, Robert W. Proctor. 2013. Asymmetry of congruency effects in spatial Stroop tasks can be eliminated. Acta Psychologica 143, 7-13. [CrossRef]

10. ROBERTO E. MERCADILLO, CELIA TRUJILLO, JULIÁN SÁNCHEZ-CORTAZAR, FERNANDO A. BARRIOS. 2012. BRAIN ACTIVITY IN ADHD PATIENTS PERFORMING THE COUNTING STROOP TASK: A SOCIAL NEUROSCIENCE APPROACH 1,2. Psychological Reports 111, 652-668. [CrossRef]

11. Guangheng Dong, Elise E. DeVito, Xiaoxia Du, Zhuoya Cui. 2012. Impaired inhibitory control in 'internet addiction disorder': A functional magnetic resonance imaging study. Psycbiatry Research: Neuroimaging 203, 153-158. [CrossRef]

12. Liam J. Nestor, Dara G. Ghahremani, John Monterosso, Edythe D. London. 2011. Prefrontal hypoactivation during cognitive control in early abstinent methamphetamine-dependent subjects. Psychiatry Research: Neuroimaging . [CrossRef]

13. Sven Vanneste, Dirk Ridder. 2011. The Use of Alcohol as a Moderator for Tinnitus-Related Distress. Brain Topography . [CrossRef] 